\title{
Finite Size Scaling of the 2D Six-Clock model
}

\author{
M. Itakura \\ Center for Promotion of Computational Science and Engineering, Japan Atomic Energy Research \\ Institute, Meguro-ku, Nakameguro 2-2-54, Tokyo 153, Japan
}

(November 13, 2018)

\begin{abstract}
We investigate the isotropic-anisotropic phase transition of the twodimensional XY model with six-fold anisotropy, using Monte Carlo renormalization group method. The result indicates difficulty of observing asymptotic critical behavior in Monte Carlo simulations, owing to the marginal flow at the fixed point.
\end{abstract}

PACS numbers: 02.70.Lq, 75.10.Hk 
The XY model with $Z_{6}$ symmetry breaking field represents not only planar spin magnets with $Z_{6}$ symmetric crystal field [1], but also models with $Z_{2} \times Z_{3}$ symmetry (which is isomorphic to $Z_{6}$ ) such as three-state antiferromagnetic Potts model on the square and the cubic lattice [2] and Ising antiferromagnet on the triangular and the stacked-triangular lattice [3 8].

The critical behavior of the model in two dimension is well understood by scaling argument [1]: The model undergoes two distinct phase transitions, both of which being Kosterlitz-Thouless transition. In Monte Carlo simulations of finite system, however, marginal renormalization flow near the fixed point makes it difficult to observe asymptotic critical behavior. In the present work, we used improved Monte Carlo renormalization group method [9] to observe the renormalization flow of the six-clock model on the square lattice of size up to $64 \times 64$, and found that the size $64 \times 64$ is still insufficient to observe asymptotic critical behavior. Similar situation occurs in several other models, such as general spin models in $D=4$ [9] and $O(3)$ spin model with cubic anisotropy in $D=3$ [10].

In the Ref. [1] by José et.al., they investigated the following model:

$$
H=K \sum_{<i j>} \cos \left(\theta_{i}-\theta_{j}\right)+A \sum_{i} \cos \left(p \theta_{i}\right)
$$

where the first summation runs over all nearest pairs on the square lattice, and $p$ is some integer. From Gaussian spin-wave theory and scaling analysis, it can be shown that the perturbation term $\sum_{i} \cos \left(p \theta_{i}\right)$ is relevant when $\eta<4 / p^{2}$, where $\eta$ is the critical exponent of long distance spin-spin correlation defined as below:

$$
<\cos \left(\theta_{x}-\theta_{y}\right)>\sim|x-y|^{-\eta} \text { for }|x-y| \gg 1 .
$$

Thus flow of the renormalized parameters $K$ and $A$ for $p=6$ case is expected to become the one shown in Fig. 1: The parameter space is divided into three regions, namely hightemperature (H), Kosterlitz-Thouless (KT), and low-temperature (L) phases. Any model whose initial parameter crosses the L-KT boundary is attracted to a fixed point which is on the KT fixed line and characterized by an exponent $\eta=1 / 9$ (we denote the fixed point as $\left.F_{1 / 9}\right)$ and exhibits KT-phase transition. Near the fixed point $F_{1 / 9}$, renormalization flow along the L-KT boundary line slows down because $F_{1 / 9}$ is marginal for both direction $K$ and $A$. Thus finite size correction (distance to the final fixed point) is expected to behave like $1 / \log L$ ( $L$ is linear size of the system) and one can not observe asymptotic critical behavior unless extremely large system is used. For example, the critical exponent $\eta$ at the lower critical temperature of Ising antiferromagnet on the triangular lattice has been estimated by several authors (to confirm theoretical prediction $\nu=1 / 9=1.111 \cdots$ ) as $\eta=0.15(2)$ [4] and $\eta=0.125(25)$ [6]. The accuracy of these values are relatively low compared to other models such as $O(n)$ spin models in three dimension: this implies that there are large finite-size correction.

In the present work we numerically investigated the renormalization flow of the model (1]) using the improved Monte Carlo Renormalization Group (MCRG) method [9], which is very simple and efficient way to extract essential information of critical phenomena from simulation data. We observed the following quantities:

$$
K_{L}=1-\frac{<\mathbf{S}\left(\mathbf{k}_{1}\right) \cdot \mathbf{S}\left(-\mathbf{k}_{1}\right)>}{<\mathbf{S}(0)^{2}>}, \quad A_{L}=\frac{<R_{M}^{6} \cos \left(6 \theta_{M}\right)>}{<R_{M}^{2}>^{3}}
$$


where

$$
\mathbf{S}(\mathbf{k}) \equiv L^{-2} \sum_{\mathbf{x}} \exp (i \mathbf{k} \cdot \mathbf{x})\left(\cos \theta_{\mathbf{x}}, \sin \theta_{\mathbf{x}}\right), \quad \mathbf{k}_{1} \equiv(2 \pi / L, 0)
$$

and

$$
\mathbf{M} \equiv \mathbf{S}(0)=R_{M}\left(\cos \theta_{M}, \quad \sin \theta_{M}\right)
$$

$K_{L}$ and $A_{L}$ reflect the behavior of the renormalized temperature and anisotropy (by a factor $L)$, respectively. We also observed Binder's parameter and $\left\langle\cos 6 \theta_{M}>\right.$, and found that $K_{L}$ and $A_{L}$ reflect the RG flow better than these quantities.

The Hamiltonian (11) on a $L \times L$ square lattice is simulated for $L=16,32$, and 64. In Monte Carlo simulation, Metropolis update scheme and Wolff's cluster algorithm [11] are combined. In the Metropolis update, we choose new spin value $\theta$ with a probability proportional to $\exp [-A \cos (6 \theta)]$ and calculate acceptance probability using only $K \sum_{<i j>} \cos \left(\theta_{i}-\theta_{j}\right)$. This scheme satisfies the detailed-balance condition of the Hamiltonian (1) and improves the acceptance ratio for large $A$. In the cluster update, spin-reflection axis is restricted to the one which preserves the anisotropy. The Hamiltonian (1) is simulated for $A=\infty, 0.5,0.2,0.1$, and 0.05 cases.

Figure 2 shows the flow of $K_{L}$ and $A_{L}$ : each line is drawn from $\left(K_{L}, A_{L}\right)$ to $\left(K_{2 L}, A_{2 L}\right)$. One can see that the obtained RG flow agrees well with the theoretical one. The position of the critical point $F_{1 / 9}$ is estimated by plotting $\log \left\langle M_{L}^{2}\right\rangle+(\log L) / 9$ versus $\log L$ for various $K$, being $A$ fixed to zero: since $<M_{L}^{2}>\sim L^{-\eta}$, the plot becomes horizontal at $F_{1 / 9}$. Fig. 3 indicates that $F_{1 / 9}$ is located near $K=1.7$. The value of $K_{L}$ at this temperature is marked as $F_{1 / 9}$ in Fig. 2. One can see that the $\mathrm{RG}$ flow is attracted to the KT line in the left (high-temperature) side of $F_{1 / 9}$, while the flow deviates from the KT line in the right (low-temperature) side of $F_{1 / 9}$. Plots for $A=\infty$ case seems to approach $F_{1 / 9}$ as $L$ increases. However, the approach is indeed slow and extremely large $L$ is required to observe convergence to $F_{1 / 9}$.

The distance to $F_{1 / 9}$ results in systematic error in the finite-size scaling analysis [4,6], such as position of the critical point and value of the critical exponent, and one can not extrapolate the $L=\infty$ limit owing to the slow vanishing correction term. For example, if one define finite-size critical point as a temperature where renormalized anisotropy becomes size-independent, it will deviate to low-temperature side as Fig. 1 suggests. Similarly, the $\eta=1 / 9$ criterion will lead to systematic error, because effective exponent for $\eta$ at $A \neq 0$ region generally differ from that of asymptotic value at $A=0$.

In conclusion, we demonstrated that it is nearly impossible, by means of Monte Carlo simulations of finite system to observe asymptotic critical behavior of isotropic-anisotropic phase transition in 2D six-clock model and those of similar symmetry. Although quantitative information such as position of critical point or values of critical exponents are hard to obtain, qualitative information such as presence or absence of the transition can be easily obtained by observing renormalized anisotropy, and it is enough for Monte Carlo simulation since the nature of the transition, such as critical exponents, is already well known. 


\section{REFERENCES}

[1] J. V. José, L. P. Kadanoff, S. Kirkpatrick and D. R. Nelson, Phys. Rev. B 16, 1217 (1977).

[2] J. S. Wang, R. H. Swendsen and R. Kotecký, Phys. Rev. Lett. 63, 109 (1989) ; Phys. Rev. B 42, 2465 (1990) and references therein.

[3] M. L. Plumer, A. Mailhot, R. Ducharme, A. Caill and H. Diep, Phys. Rev. B 47, 14312 (1993) and references therein.

[4] D. P. Landau, Phys. Rev. B 27, 5604 (1983).

[5] H. Kitatani and T. Oguchi, J. Phys. Soc. Jpn. 57, 1344 (1988).

[6] S. Miyashita, H. Kitatani and Y. Kanada, J. Phys. Soc. Jpn. 60, 1523 (1991).

[7] S. L. A. de Queiroz and E. Domany, Phys. Rev. E 52, 4768 (1995) and references therein.

[8] S.Miyashita, J. Phys. Soc. Jpn. 66, 3411 (1997).

[9] M.Itakura, Phys. Rev. E 61, 5924 (2000).

[10] M. Caselle and M. Hasenbusch, J. Phys. A 31, 4603 (1998).

[11] U. Wolff, Phys. Rev. Lett. 62, 361 (1989). 


\section{FIGURES}

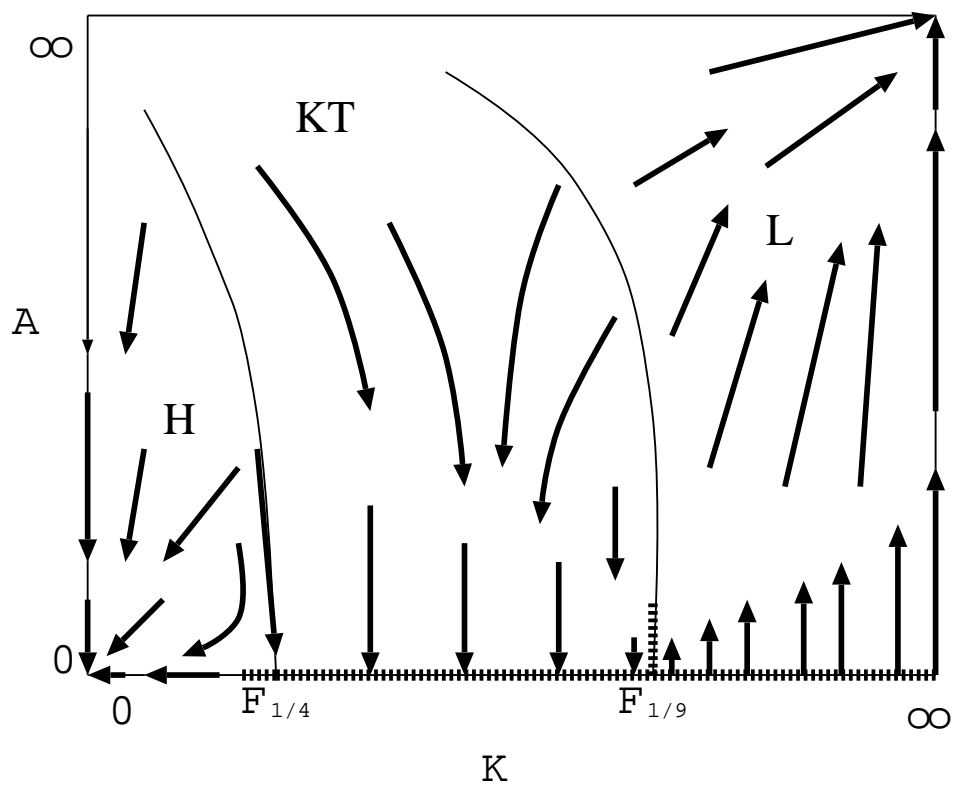

FIG. 1. Theoretical prediction of the renormalization flow of temperature and anisotropy. L, $\mathrm{KT}$, and $\mathrm{H}$ denotes low-temperature, Kosterlitz-Thouless, and high-temperature phase, respectively. Dotted lines indicate direction of marginal renormalization flow.

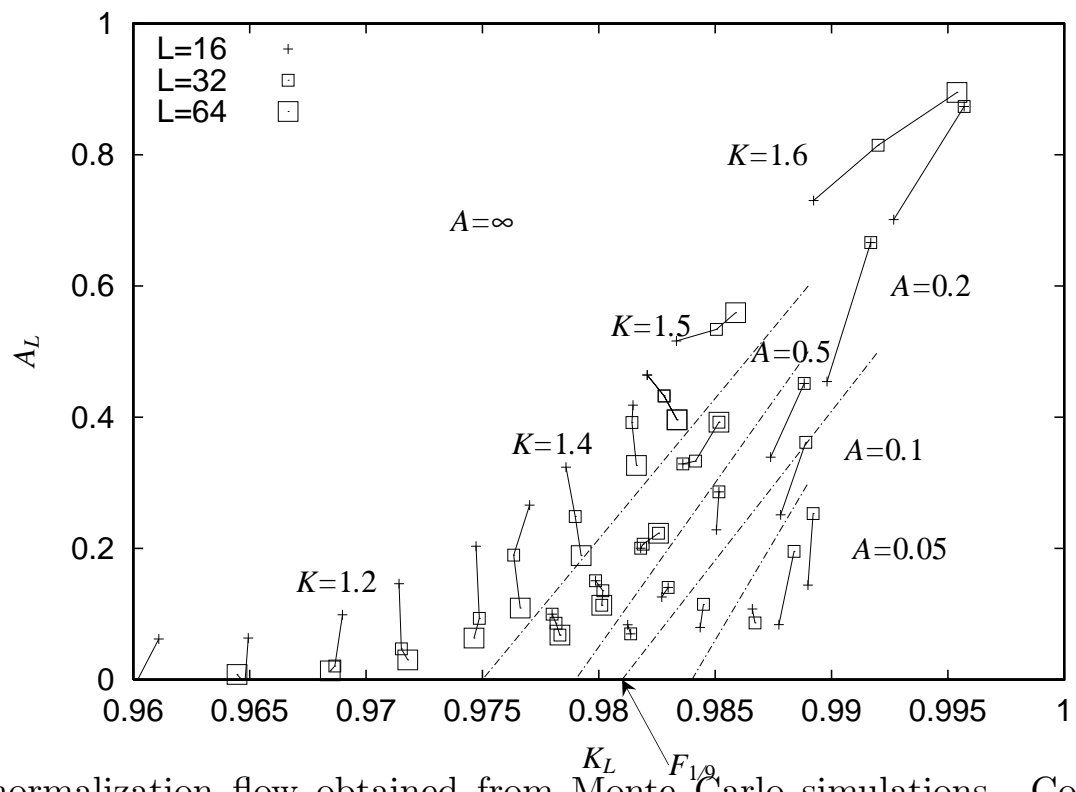

FIG. 2. Renormalization flow obtained from Monte Carlo simulations. Connected two line segments are drawn from $L=16$ data to $L=32$ data, then to $L=64$ data. Other single lines are drawn from $L=16$ data to $L=32$ data. Plots for each different values of $A$ are separated by dotted lines. The position of $F_{1 / 9}$ was estimated from finite-size scaling of $\left\langle M_{L}^{2}\right\rangle$. 


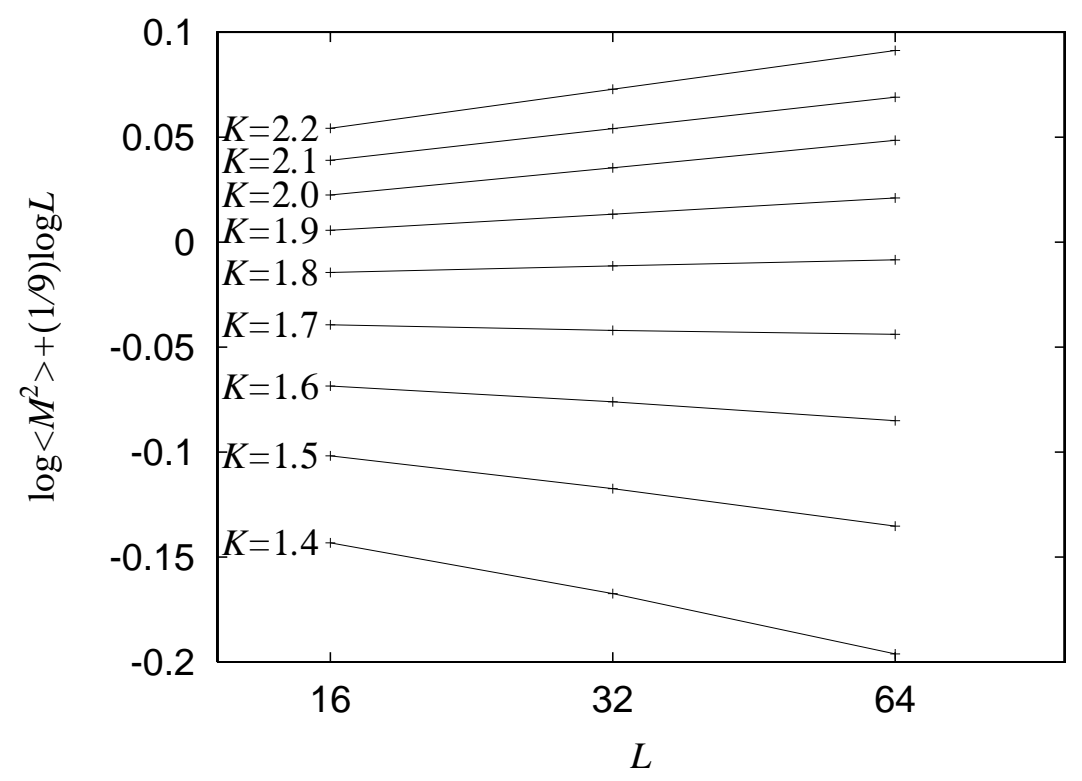

FIG. 3. Plot of $\log <M_{L}^{2}>+\frac{1}{9} \log L$ versus $\log L$ for $A=0$. 